\title{
Erratum to: Hyperbolic Distortion, Boundary Behaviour and Finite Blaschke Products
}

\author{
Nina Zorboska $\mathbf{1}^{1}$
}

Published online: 25 March 2015

(C) Springer-Verlag Berlin Heidelberg 2015

\section{Erratum to: Comput. Methods Funct. Theory DOI 10.1007/s40315-014-0099-2}

This errata note is a correction to Proposition 2.2 in the original version of the paper. We provide here a corrected statement of the result with its corrected proof. The proposition requires an additional assumption of univalence, since the proof uses a result from [1, p. 71] which requires it. Note that the result in [1, p. 71] does require the univalence, even though it has been stated there without this assumption.

Proposition 2.2 Let $\phi$ be a univalent self-map of $\mathbb{D}$, and let $\zeta \in \partial \mathbb{D}$ be such that $\lim _{z \rightarrow \zeta} \tau_{\phi}(z)=0$. Then there exists $\Gamma$, an open subarc of $\partial \mathbb{D}$ containing the point $\zeta$, such that the only possible subsets of $\Gamma$ mapped by $\phi$ into $\partial \mathbb{D}$ are sets of measure zero.

Proof Since $\lim _{z \rightarrow \zeta} \tau_{\phi}(z)=0$, for any $0<\epsilon<1, \exists \delta>0$ such that $\tau_{\phi}(z)<\epsilon$ whenever $z \in B(\zeta, \delta) \cap \mathbb{D}$. Let $\Gamma_{\delta}=B(\zeta, \delta) \cap \partial \mathbb{D}$. Then $\forall \xi \in \Gamma_{\delta}$ we have that

$$
\angle \lim _{z \rightarrow \xi} \tau_{\phi}(z) \leq \epsilon<1
$$

and so $\phi$ can not have an angular derivative at any $\xi \in \Gamma_{\delta}$.

Let $E=\left\{\xi \in \Gamma_{\delta}: \phi(\xi) \in \partial \mathbb{D}\right\}$, and let $\psi$ be the inverse to the Riemann mapping from $B(\zeta, \delta) \cap \mathbb{D}$ onto $\mathbb{D}$. By the Carathéodory's extension theorem [2, p. 24], $\psi$ can

Communicated by Elias Wegert.

The online version of the original article can be found under doi:10.1007/s40315-014-0099-2.

$\bowtie \quad$ Nina Zorboska

zorbosk@cc.umanitoba.ca

1 Department of Mathematics, University of Manitoba, Winnipeg, MB R3T 2N2, Canada 
be extended to a homeomorphism between $\overline{\mathbb{D}}$ and $\overline{B(\zeta, \delta) \cap \mathbb{D}}$. Let $\varphi=\phi \circ \psi$. Then $\varphi$ is a univalent self-map of $\mathbb{D}$ and $\psi^{-1}(E)$ is a subset of $\partial \mathbb{D}$ on which $\varphi$ has radial limits of modulus one.

The proof of a result from [1, p. 71] shows that a univalent self-map of $\mathbb{D}$ with radial limits of modulus one on a set of positive measure must have an angular derivative at some point of $\partial \mathbb{D}$. Thus, if $E$ is of positive measure, since by the Riesz-Privalov theorem [2, p. 126] $\psi^{-1}(E)$ is also of positive measure, $\varphi$ must have an angular derivative at some point $w$ of $\partial \mathbb{D}$. Note that then $w \in \psi^{-1}\left(\Gamma_{\delta}\right)$, and since $\psi$ also has an analytic extension across $\psi^{-1}\left(\Gamma_{\delta}\right)$, we get that $\phi$ must have an angular derivative at some point of $\Gamma_{\delta}$. But that is not possible, and so it must be that the measure of $E$ is zero.

We don't know at this point if the result in Proposition 2.2 might still hold in the more general case when $\phi$ is not necessarily univalent.

The only place were Proposition 2.2 was used in the original paper was in the proof of Proposition 3.1, and so this correction does not affect any of the other results.

Also, as stated in the original paper, the result in Proposition 3.1 is a well known fact that the only inner functions with bounded $\alpha$-hyperbolic local distortion, $0<\alpha<1$, are the finite Blaschke products. This is true since the corresponding $\alpha$-Bloch-type spaces are Lipschitz spaces of order $1-\alpha$, which are further contained in the disk algebra (see [3, p. 74]). Thus, the only inner functions that belong to an $\alpha$-Bloch-type space with $0<\alpha<1$ are the finite Blaschke products. Our original intention was to provide a different proof of this fact by using Proposition 2.2. Since an univalent inner function is a disk-automorphism, i.e. already a finite Blaschke product, Proposition 3.1 is trivial for univalent inner functions, and so, its alternative proof using Proposition 2.2 is obsolete.

\section{References}

1. Shapiro, J.H.: Composition Operators and Classical Function Theory. Springer, New York (1993)

2. Pommerenke, C.: Boundary Behaviour of Conformal Maps. Springer, Berlin (1992)

3. Duren, P.L.: Theory of $H^{p}$ Spaces, vol. 38. Academic Press, New York (1970) 